\title{
Investigating the Numerical Solution of the BoltzmannTransport Equation in Silicon in Momentum Space Using Computational Systems of Different Dimensions
}

\author{
Mahmood Majed Mahmood ${ }^{1 *}$, Mumtaz M. Hussien² \\ ${ }^{1,2}$ Department of Physics, College of Education for Pure Sciences, University of Mosul, Mosul, Iraq \\ E-mail: ${ }^{1 *}$ mahmood.majed.mahmood@gmail.com, ${ }^{2}$ momtaz hussien@uomosul.edu.iq
}

(Received March 24, 2020; Accepted July 22, 2020; Available online December 01, 2020)

DOI: 10.33899/edusj.2020.126828.1057, (@ 2020, College of Education for Pure Science, University of Mosul.

This is an open access article under the CC BY 4.0 license (http://creativecommons.org/licenses/by/4.0/).

\begin{abstract}
:
The Boltzmann transport equation is the basic equation for solving the transport of charge carrier (electrons, holes) problems in semiconductor devices. The distribution function has been obtained from the solution of this equation. The distribution function is important in calculating semiconductor properties, which can be used to calculate the average electron energy, the charge carrier concentration, and other properties. In this work the semi-classical Boltzmann transport equation in silicon was solved using analytical / numerical methods in steady state case in momentum space. The analytical solution is requires expressing the distribution function using Legendre polynomials expansion the first two terms of the expansion, by taking into account the effect of both acoustic elastic scattering and nonelastic scattering in addition to the effect of non-parabolic energy band structure. In order to obtain the numerical solution of Boltzmann transport equation the finite difference method is used. The differential equation is transformed to linear difference equation which can represented by matrices. Numerical systems with different dimensions are designed to calculate the distribution function with the least possible time to maintain the accuracy of the solution for different applied electric field which represent the low and high field regions at temperatures $\mathrm{T}=(77,300) \mathrm{K}$. The obtained results showed good agreement with published data that used other calculation methods such as the Monte Carlo simulation method for all the system used in this work.
\end{abstract}

Keyword: Boltzmann equation in Silicon, numerical solution for Boltzmann transport equation, Legendre polynomial expansion method

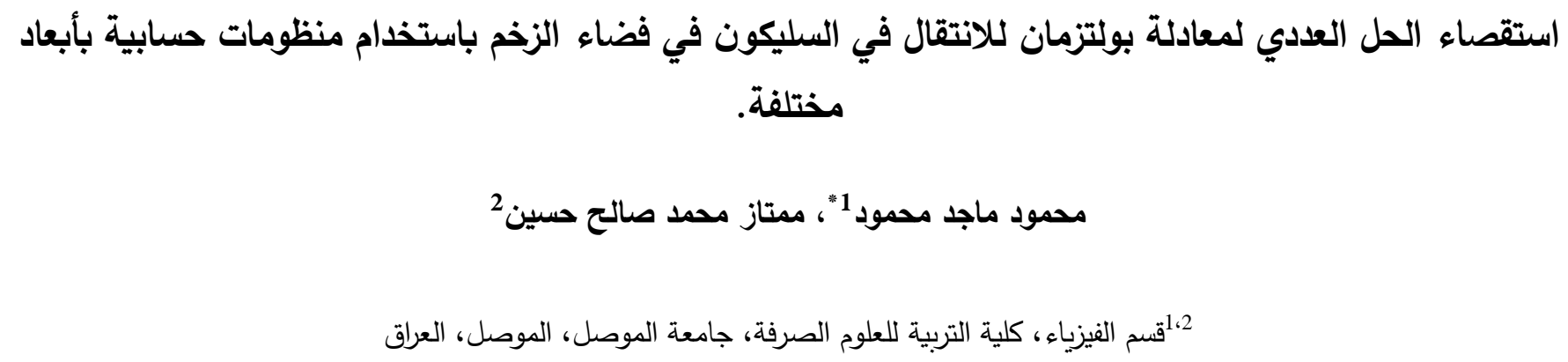




\section{Journal of Education and Science (ISSN 1812-125X), Vol: 29, No: 4, 2020 (108-123)}

الملخص:

تعد معادلة الانتقال لبولتزمان المعادلة الاساسية المستخدمة لحل مسائل انتقال حامل الثحنة (الالكترون، الفجوة) في نبائط أشباه الموصلات ويمكن الحصول على دالة التوزيع من خلال حل هذه المعادلة، تعد دالة التوزيع مهمة في حساب خصائص أشباه الموصلات، التي يمكن استخدمها في حساب متوسط طاقة الإكترون وتركيز حامل الثحنة وغيرها من الخواص. في هذا البحث تم حل معادلة الانتقال شبه الكلاسيكية لبولتزمان في السليكون باستخدام طريقة تحليلية/عددية في حالة الاستقرار في فضاء الزخم. يتطلب الحل التحليلي التعبير عن دالة التوزيع باستخدام متعددات حدود لاجندر وذلك بأخذ الحدين الاول والثاني من امتدادات متعددات لاجندر مع الاخذ بنظر الاعتبار تأثير كل من الاستطارة السمعية المرنة و الاستطارة غير المرنة إضافة الى تأثير تركيب حزمة الطاقة غير المكافئ. وللحصول على الحل العددي لمعادلة بولتزمان نستخدم طريقة الفروق المحدة اذ يتم تحويل المعادلة التفاضلية إلى معادلة الفروق الخطية التي يمكن تمثيلها بالمصفوفات. تم تصميم عدة منظومات عددية بأبعاد مختلفة لحساب دالة التوزيع لكل منظومة، وتمت المقارنة بين هذه المنظومات من حيث الدقة ولمجالات كهربائية مسلطة مختلفة تمتد من منطقة المجالات الواطئة الى منطقة المجالات العالية وعند درجتي الحرارة K (77, 300)، وأظهرت النتائج التي تم الحصول عليها توافقا جيد مع النتائج المنشورة التي استخدمت طرق اخرى مثل طريقة محاكاة

$$
\text { مونتي كارلو ولمعظم المنظومات المستخدمة في البحث. }
$$

الكلمات الدالة: معادلة بولتزمان في السليكون، الحل العددي لمعادلة الانتقال لبولتزمان، طريقة متعددات حدود لاجندر .

\section{المقدمة}

تظهر اهمية دراسة دالة التوزيع في تصميم نبائط اشباه الموصلات وتطبيقاتها الاكترونية مثل ترانزستور تأثير المجال، اذ تقدم لنا دالة التوزيع احتمالية ايجاد حامل الثحنة(الالكترونات والفجوات) ضمن متجه موجة معين في فضاء الزخم الأكتروني [1]. تتوعت طرائق حل معادلة الانتقال لبولتزمان الى طرائق تحليلية اذ تم افتراض ان لدالة التوزيع توزيعا اسيا او توزيعاً ماكسويلياً [2,3]. اذ الطريقة التحليلية ذات نتائج جيدة الا انها تكون مقتصرة على نماذج فيزيائية محدة ضمن مدى محدود للطاقة [1]. وكذلك لا يمكن استخدامها لبعض اشباه الموصلات مثل ارسنايد الكاليوم GaAs ولا يصح استخدامها لجميع اشباه الموصلات عند المجالات العالية [4]. تم التوجه نتيجة لذلك من قبل الباحثين لاستخدام طرائق عددية لحل معادلة الانتقال لبولتزمان وحصاب دالة التوزيع مثل طريقة محاكاة مونتي كارلو التي تعتمد على الوقت المستغرق لحساب دالة التوزيع [5,6,7]. تم تقديم طريقة تحليلية-عددية ومن اجل تقليل الوقت يتضمن الجانب التحليلي في هذه الطريقة تمثيل دالة التوزيع كدالة لاجندر التي تضم جزء متتاظراً وأخر غير متناظر اما الجانب العددي فيتم استخدام معادلات الفروق لتحويل المعادلات الرياضية الى مصفوفة بعد التعويض عن قيم مختلفة ومدى محدد من الطاقة [8].

مع تطور طرائق تصنيع النبائط الاككترونية وتصغير حجمها الى الابعاد النانوية تطلب وضع نموذج جديد يتتاسب مع القوانين الفيزيائية في عالم النانو، من هذه النماذج هو نموذج الهيدروداينمك الموسع الذي يعتمد على ميكانيك الكم ومعادلة شرودينكر [9]. استخدم هذا النموذج في حساب دالة التوزيع للإلكترون وحساب معلمات الانتقال لحامل الثحنة في اسلاك نانوية من السليكون [10]. 
في هذا البحث تم تصميم برنامج حاسوبي لحساب دالة التوزيع للإلكترون في السليكون اعتمادا على حل معادلة الانتقال لبولتزمان شبه الكلاسيكية. اذ استطعنا حل معادلة الانتقال لبولتزمان شبه الكلاسيكية باستخدام طريقة تحليلية - عددية وحساب دالة التوزيع للاكترون في السليكون لدرجتي حرارة K(77-300) مع الاخذ بنظر الاعتبار تاثير كل من الاستطارة السمعية المرنة والاستطارة غير المرنة بنوعيها السمعية والبصرية وكذلك تأثير تركيب حزمة الطاقة ذات القطع غير المكافئ ولمدى طاقة eV-2(0)، وتمكنا ايضا من التحكم بابعاد المصفوفة وتقليل الزمن المستغرق في حساب دالة التوزيع.

الجزء النظري:

\section{معادلة الانتقال لبولتزمان شبه الكلاسيكية}

تعد معادلة الانتقال لبولتزمان شبه الكلاسيكية معادلة تفاضلية - تكاملية تطبق عليها قوانين نيوتن وقوانين ميكانيك الكم [4]. اذ

يمكن اعادة صياغة هذه المعادلة, مع الاخذ بنظر الاعتبار تأثير الاستطارة السمعية المرنة و الإستطارة غير المرنة وكذلك تأثير المجال الكهربائي وتركيب حزمة الطاقة ذات القطع غير المكافئ, من اجل حساب دالة التوزيع للإلكترون في السليكون. يمكن كتابة معادلة الانتقال القياسية عند حالة الاتزان في فضاء الزخم بالصيغة الاتية [4]:

$\frac{\partial f}{\partial t}+v \cdot \nabla_{r} f+F \cdot \nabla_{p} f=\left.\frac{\partial f}{\partial t}\right|_{c o l l}+s(r, p, t)$

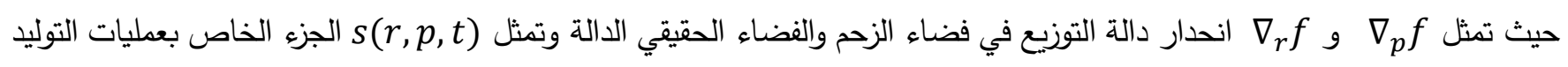
واعادة الاتحاد ويمثل الحد ويسى الحد (

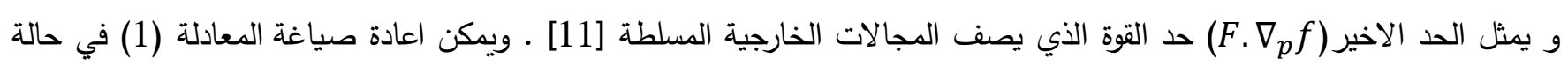
الاستقرار وعند عدم وجود عمليات التوليد واعادة الاتحاد بالشكل الاتي:

$-\frac{e \vec{E}}{\hbar} \cdot \vec{\nabla}_{\vec{k}} f(k)=\left(\frac{\partial f}{\partial t}\right)_{a c}+\left(\frac{\partial f}{\partial t}\right)_{i v}$

اذ ان E تمثل المجال الكهربائي الثابت الذي تم اخذه بالاتجاه البلوري[111] لتبسيط الحل اذ يمثل هذا الاتجاه من اتجاهات التناظر العالي، حد الإستطارة غير المرنة (أي الاستطارة بين منخفضات الطاقة المختلفة). حل معادلة الانتقال شبه الكلاسيكية لبولتزمان: 
يمكن حل معادلة الانتقال لبولتزمان المتجانسة بعدة طرائق منها تحليلية مثل طريقة تقريب زمن الاسترخاء واخرى عددية مثل طريقة مونتي كارلو. ولصعوبة الحل استخدمت طرائق اخرى ناتجة من جمع الطريقة التحليلية مع الطريقة العددية. تم في هذا البحث استخدام طريقة تحليلية لتمثيل دالة التوزيع بدالة متعددة حدود لاجندر وطريقة عددية متمثلة بمعادلات الفروق [8,12].

\section{الحل التحليلي لمعادلة الانتقال لبولتزمان:}

لتبسيط حل معادلة الانتقال لبولتزمان باستخدام متعددة حدود لاجندر وجعلها قابلة للحل هناك مجموعة من التقريبات التي يجب القيام بها. التقريب الاول هو تحويل سطوح الطاقة الثابتة البيضوية للسليكون الى سطوح كروية باستخدام تقريب هيرنك - فوجت وبتطبيق هذا التحويل يصبح من الممكن العمل في الفضاء النجمي لمتجه الزخم (الفضاء النجمي لمتجه موجة الاككترون k*- space [5]. تعطى العلاقة بين متجه الموجة في الفضاء النجمي والفضاء الحقيقي بالمعادلة الاتية:

$$
\vec{k}^{*}=\hat{\alpha}^{1 / 2} \vec{k}
$$

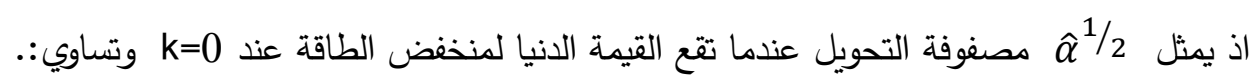

$\hat{\alpha}^{1 / 2}=\left(\begin{array}{ccc}\left(\frac{m_{o}}{m_{x}}\right)^{1 / 2} & 0 & 0 \\ 0 & \left(\frac{m_{o}}{m_{y}}\right)^{1 / 2} & 0 \\ 0 & 0 & \left(\frac{m_{o}}{m_{z}}\right)^{1 / 2}\end{array}\right)$

تمثل التوالي. اذا كانت سطوح الطاقة ذات شكل بيضوي فان مصفوفة التحويل الى سطوح طاقة ذات شكل كروي لاي منخفض طاقة تعطى بالعلاقة الاتية: تكون الصيغة الاتية:

$$
\hat{\alpha}^{1 / 2}=\left(\begin{array}{ccc}
\left(\frac{m_{o}}{m_{t}}\right)^{1 / 2} & 0 & 0 \\
0 & \left(\frac{m_{o}}{m_{t}}\right)^{1 / 2} & 0 \\
0 & 0 & \left(\frac{m_{o}}{m_{l}}\right)^{1 / 2}
\end{array}\right)
$$


تمثل $m_{l}$ و $m_{t}$ الكتلة الفعالة للالكترون في الاتجاهين الطولي والمستعرض على التو الي. يمكن حساب العلاقة بين متجه الموجة والطاقة ع باستخدام قانون كاين للتفريق [8,9].

$$
\gamma(\varepsilon)=\varepsilon(1+\alpha \varepsilon)=\frac{\hbar^{2} k^{* 2}}{2 m^{*}}
$$

اذ ان $\alpha$ هو معامل التحويل ووحدة قياسه (1/eV) يمكن حسابه من المعادلة.

$\alpha=\frac{\left(1-\frac{m^{*}}{m_{o}}\right)^{2}}{\varepsilon_{g}}$

اذ تمثل وع فجوة الطاقة لثبه الموصل. ويتم تحويل المجال الكهربائي من الفضاء الحقيقي الى الفضاء النجمي للتجه موجة الاككترون

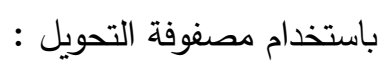

$\boldsymbol{E}^{*}=\hat{\alpha}^{1 / 2} \boldsymbol{E}$

بعد اجراء التحويل الى الفضاء النجمي لمتجه الموجة النجمي يمكن كتابة علاقة التقريق معادلة (6) حيث يهمل حد الانتشار في معادلة

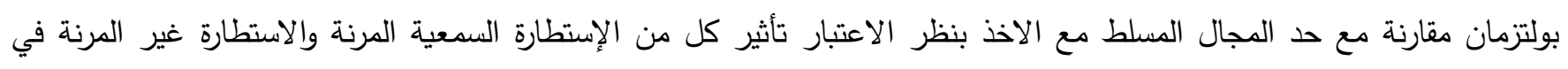

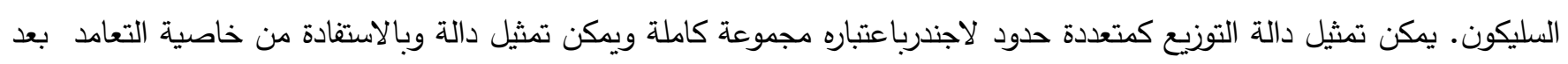
اكمال عمليات التقريب بالثكل الاتي [1]:

$f\left(\vec{k}^{*}\right)=\sum_{l=0}^{\infty} f_{l}\left(k^{*}\right) P_{l}(\cos \theta)$

بأخذ الحدين الاول والثاني من المعادلة (9) اذ يمثل الحد الاول الجزء المتماثل من دالة التوزيع ويمثل الحد الثاني الجزء غير المتماثل اذ يمكن تمثيل دالة موجة الالكترون في الفضاء النجمي بالمعادلة الاتية:

$f\left(\vec{k}^{*}\right)=f_{o}(\varepsilon)+k^{*} g(\varepsilon)(\cos \theta)$

الزاوية $\theta$ الزاوية بين الاككترون والمجال المسلط و (ع) التوزيع و (ع) و معامل لجندر من المرتبة الاولى و $\cos$ هو دالة لاجندر من المرتبة الاولى، بعد تعويض المعادلة (10) في المعادلة (2) نحصل على المعادلة الاتية:

$-\frac{e \vec{E}}{\hbar} \cdot \vec{\nabla}_{\vec{k}}\left[f_{o}(\varepsilon)+k^{*} g(\varepsilon)(\cos \theta)\right]=\left(\frac{\partial f}{\partial t}\right)_{a c}+\left(\frac{\partial f}{\partial t}\right)_{i v}$

تمثل الجهة اليسرى من المعادلة (11) حد المجال الكهربائي والذي يمكن كتابته بالصيغة الاتية:

$\left[\frac{\partial f\left(k^{*}\right)}{\partial t}\right]_{f l d}=-\frac{e}{\hbar} E^{*} \cdot \nabla_{k^{*}}\left[f_{o}(\varepsilon)+k^{*} g(\varepsilon)(\cos \theta)\right]$ 


$$
\begin{aligned}
& \text { يصبح حد المجال الكهربائي بعد مجموعة من العمليات الجبرية بالثكل الاتي: } \\
& {\left[\frac{\partial f(\varepsilon)}{\partial t}\right]_{f l d}=-\frac{e^{2} E^{* 2} \tau_{o}}{m_{o}}\left\{\left[\frac{2}{3 \gamma^{1 / 2}(\varepsilon) \gamma^{\prime 2}(\varepsilon)}-\frac{4 \gamma^{1 / 2}(\varepsilon) \gamma^{\prime \prime}(\varepsilon)}{3 \gamma^{\prime 4}(\varepsilon)}\right] \frac{\partial f_{o}(\varepsilon)}{\partial \varepsilon}+\frac{2 \gamma^{1 / 2}(\varepsilon)}{3 \gamma^{\prime 3}(\varepsilon)} \frac{\partial^{2} f_{o}(\varepsilon)}{\partial \varepsilon^{2}}\right\}} \\
& {\left[\frac{\partial f_{o}}{\partial t}\right]_{c o l l}=\left[\frac{\partial f_{o}}{\partial t}\right]_{i v}+\left[\frac{\partial f_{o}}{\partial t}\right]_{a c}=\sum_{j=1}^{15} \frac{D_{i v}^{2}\left(m_{t}^{2} m_{l}\right)^{1 / 2}}{\sqrt{2} \pi \hbar^{3} \rho \omega_{j}}\left(e^{\frac{\hbar \omega_{j}}{k_{\beta} T}}-1\right)^{-1}} \\
& \times\left\{\gamma^{1 / 2}\left(\varepsilon+\hbar \omega_{j}\right) \gamma^{\prime}\left(\varepsilon+\hbar \omega_{j}\right) \times\left[e^{\frac{\hbar \omega_{j}}{k^{T}}} \times f_{0}\left(\varepsilon+\hbar \omega_{j}\right)-f_{0}(\varepsilon)\right]+\gamma^{1 / 2}\left(\varepsilon-\hbar \omega_{j}\right) \gamma^{\prime}\left(\varepsilon-\hbar \omega_{j}\right) \times\right. \\
& \left.\left[f_{0}\left(\varepsilon-\hbar \omega_{j}\right)-e^{\frac{\hbar \omega_{j}}{k^{T} T}} f_{0}(\varepsilon)\right]\right\} \quad+\frac{4 \sqrt{2} D_{a c}^{2} m_{t}^{2} m_{l}^{1 / 2}}{\pi \hbar^{4} \rho} \gamma^{1 / 2}(\varepsilon) \gamma^{\prime 2}(\varepsilon) \times\left\{\left[\frac{\gamma(\varepsilon) k_{\beta} T}{2 \gamma^{\prime}(\varepsilon)}\right] \frac{\partial^{2} f_{0}(\varepsilon)}{\partial \varepsilon^{2}}+\left[\frac{\gamma(\varepsilon)}{2 \gamma^{\prime}(\varepsilon)}+k_{\beta} T \times\right.\right. \\
& \left.\left.\left(\begin{array}{c}
1+ \\
\gamma^{\prime 2}(\varepsilon) \gamma^{\prime \prime}(\varepsilon)
\end{array}\right)\right] \times \frac{\partial f_{0}(\varepsilon)}{\partial \varepsilon}+\left[\left(1+\frac{\gamma(\varepsilon) \gamma^{\prime \prime}(\varepsilon)}{\gamma^{\prime 2}(\varepsilon)}\right)\right] \times f_{0}(\varepsilon)\right\}
\end{aligned}
$$

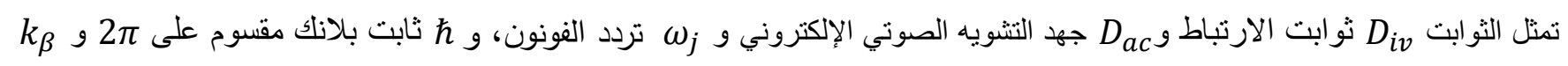
ثابت بولنزمان و م الكثافة وتصبح المعادلة النهائية عند مساواة المعادلتين (14,13) بالثكل الاتي:

$$
\begin{aligned}
& -\frac{e^{2} \tau_{0} E^{* 2}}{m_{0}}\left\{\begin{array}{c}
\left(\frac{2}{3 \gamma^{1 / 2}(\varepsilon) \gamma^{\prime 2}(\varepsilon)}-\frac{4 \gamma^{1 / 2}(\varepsilon) \gamma^{\prime \prime}(\varepsilon)}{3 \gamma^{\prime 4}(\varepsilon)}\right) \frac{\partial f_{0}(\varepsilon)}{\partial \varepsilon} \\
+\frac{2 \gamma^{1 / 2}(\varepsilon)}{3 \gamma^{\prime 3}(\varepsilon)} \frac{\partial^{2} f_{0}(\varepsilon)}{\partial \varepsilon^{2}}
\end{array}\right\}=\sum_{j=1}^{15} \frac{D_{i v}^{2}\left(m_{t}^{2} m_{l}\right)^{1 / 2}}{\sqrt{2} \pi \hbar^{3} \rho \omega_{j}}\left(e^{\left.\frac{\hbar \omega_{j}}{k_{\beta^{T}}}-1\right)^{-1} \times}\right. \\
& \left\{\begin{array}{c}
\gamma^{1 / 2}\left(\varepsilon+\hbar \omega_{j}\right) \gamma^{\prime}\left(\varepsilon+\hbar \omega_{j}\right)\left[e^{\frac{\hbar \omega_{j}}{k_{\beta^{T}}}} f_{0}\left(\varepsilon+\hbar \omega_{j}\right)-f_{0}(\varepsilon)\right] \\
\left.+\gamma^{1 / 2}\left(\varepsilon-\hbar \omega_{j}\right) \gamma^{\prime}\left(\varepsilon-\hbar \omega_{j}\right) \times\left[f_{0}\left(\varepsilon-\hbar \omega_{j}\right)-e^{\frac{\hbar \omega_{j}}{k_{\beta} T}} f_{0}(\varepsilon)\right]\right\} \\
+\frac{4 \sqrt{2} D_{a c}^{2} m_{t}^{2} m_{l}^{1 / 2}}{\pi \hbar^{4} \rho} \gamma^{1 / 2}(\varepsilon) \gamma^{\prime 2}(\varepsilon)\left\{\left[\frac{\gamma(\varepsilon) k_{\beta} T}{2 \gamma^{\prime}(\varepsilon)}\right] \frac{\partial^{2} f_{0}(\varepsilon)}{\partial \varepsilon^{2}}+\left[\frac{\gamma(\varepsilon)}{2 \gamma^{\prime}(\varepsilon)}+k_{\beta} T\left(1+\frac{\gamma(\varepsilon) \gamma^{\prime \prime}(\varepsilon)}{\gamma^{\prime 2}(\varepsilon)}\right)\right] \frac{\partial f_{0}(\varepsilon)}{\partial \varepsilon}\right. \\
+\left[\left(1+\frac{\gamma(\varepsilon) \gamma^{\prime \prime}(\varepsilon)}{\gamma^{\prime 2}(\varepsilon)}\right)\right] f_{0}(\varepsilon)
\end{array}\right\}
\end{aligned}
$$

تمثل المعادلة (15) الجزء المتتاظر من دالة التوزيع وهي معادلة تفاضلية من الرتبة الثانية [12]. 
تمثل المعادلة (15) معادلة تفاضلية/ فروق finite difference equation تمثل الجزء المتناظر من دالة التوزيع من الصعوبة حلها بالطرائق التحليلية لوحدها ومن اجل التغلب على هذه الصعوبات تم حل هذه المعادلة عدديا وذلك بتحويلها الى معادلة فروق اذ يمكن حلها باستخدام الطرق التكرارية او بطريقة الحذف لكاوس [13]. اذ يتم تقسيم الحل الى منظومة من مجموعة من النقاط ومن ثم حلها باستخدام طرائق المصفوفات matrix methods. يمكن تبسط المعادلة (15) باستخدام معادلات الفروق:

$\frac{\partial f_{0}(\varepsilon)}{\partial \varepsilon}=\frac{f_{i+1}-f_{i-1}}{2 \Delta \varepsilon}$

$\frac{\partial^{2} f_{0}(\varepsilon)}{\partial \varepsilon^{2}}=\frac{f_{i+1}-2 f_{i}+f_{i-1}}{(\Delta \varepsilon)^{2}}$

هنا عه مقدار الزيادة في الطاقة اما f فهي قيم (ع) $f_{0}$ عند العقد، تم عزل الحدود المتثابهة في المعادلة (15) وتبسيطها من خلال مجموعة من العمليات الجبرية:

$A_{i+1} f_{i+1}+A_{i-1} f_{i-1}+A_{i} f_{i}+A_{i+n j} f_{i+n j}+A_{i-n j} f_{i-n j}=0$

تمثل عناصر المصفوفة A على التوالي ويمكن تمثيلها بالمعادلات الاتية:

$A_{i+1}=\frac{e^{2} \tau_{0} E^{* 2}}{m_{0}}\left(\frac{2}{3 \gamma_{i}^{1 / 2} \gamma_{i}^{\prime 2}}-\frac{4 \gamma_{i}^{1 / 2} \gamma_{i}^{\prime \prime}}{3 \gamma_{i}^{\prime 4}}\right)\left(\frac{1}{2 \Delta \varepsilon}\right)+\frac{e^{2} \tau_{0} E^{* 2}}{m_{0}}\left(\frac{2 \gamma_{i}^{1 / 2}}{3 \gamma_{i}^{\prime 3}}\right)\left(\frac{1}{(\Delta \varepsilon)^{2}}\right)+$

$\frac{4 \sqrt{2} D_{a c}^{2} m_{t}^{2} m_{l}^{1 / 2}}{\pi \hbar^{4} \rho} \gamma_{i}^{1 / 2} \gamma_{i}^{\prime 2}\left[\frac{\gamma_{i} k_{\beta} T}{2 \gamma_{i}^{\prime}}\right]\left(\frac{1}{(\Delta \varepsilon)^{2}}\right)+\frac{4 \sqrt{2} D_{a c}^{2} m_{t}^{2} m_{l}^{1 / 2}}{\pi \hbar^{4} \rho} \gamma_{i}^{1 / 2} \gamma_{i}^{\prime 2}\left[\frac{\gamma_{i}}{2 \gamma_{i}^{\prime}}+k_{\beta} T\left(1+\frac{\gamma_{i} \gamma_{i}^{\prime \prime}}{\gamma_{i}^{\prime 2}}\right)\right]\left(\frac{1}{2 \Delta \varepsilon}\right)$

$A_{i-1}=-\frac{e^{2} \tau_{0} E^{* 2}}{m_{0}}\left(\frac{2}{3 \gamma_{i}^{1} / 2 \gamma_{i}^{\prime 2}}-\frac{4 \gamma_{i}^{1 / 2} \gamma_{i}^{\prime \prime}}{3 \gamma_{i}^{\prime 4}}\right)\left(\frac{1}{2 \Delta \varepsilon}\right)+\frac{e^{2} \tau_{0} E^{* 2}}{m_{0}}\left(\frac{2 \gamma_{i}^{1 / 2}}{3 \gamma_{i}^{\prime 3}}\right)\left(\frac{1}{(\Delta \varepsilon)^{2}}\right)+$

$\frac{4 \sqrt{2} D_{a c}^{2} m_{t}^{2} m_{l}^{1 / 2}}{\pi \hbar^{4} \rho} \gamma_{i}^{1 / 2} \gamma_{i}^{\prime 2}\left[\frac{\gamma_{i} k_{\beta} T}{2 \gamma_{i}^{\prime}}\right]\left(\frac{1}{(\Delta \varepsilon)^{2}}\right)-\frac{4 \sqrt{2} D_{a c}^{2} m_{t}^{2} m_{l}^{1 / 2}}{\pi \hbar^{4} \rho} \gamma_{i}^{1 / 2} \gamma_{i}^{\prime 2}\left[\frac{\gamma_{i}}{2 \gamma_{i}^{\prime}}+k_{\beta} T\left(1+\frac{\gamma_{i} \gamma_{i}^{\prime \prime}}{\gamma_{i}^{\prime 2}}\right)\right]\left(\frac{1}{2 \Delta \varepsilon}\right)$

$A_{i}=-2 \frac{e^{2} \tau_{0} E^{* 2}}{m_{0}}\left(\frac{2 \gamma_{i}^{1 / 2}}{3 \gamma_{i}^{\prime 3}}\right)\left(\frac{1}{(\Delta \varepsilon)^{2}}\right)-2 \frac{4 \sqrt{2} D_{a c}^{2} m_{t}^{2} m_{l}^{1 / 2}}{\pi \hbar^{4} \rho} \gamma_{i}^{1 / 2} \gamma_{i}^{\prime 2}\left[\frac{\gamma_{i} k_{\beta} T}{2 \gamma_{i}^{\prime}}\right]\left(\frac{1}{(\Delta \varepsilon)^{2}}\right)$

$$
\begin{aligned}
& +\frac{4 \sqrt{2} D_{a c}^{2} m_{t}^{2} m_{l}^{1 / 2}}{\pi \hbar^{4} \rho} \gamma_{i}^{1 / 2} \gamma_{i}^{\prime 2}\left(1+\frac{\gamma_{i} \gamma_{i}^{\prime \prime}}{\gamma_{i}^{\prime 2}}\right) \\
& -\sum_{j=1}^{15} \frac{D_{i v}^{2}\left(m_{t}^{2} m_{l}\right)^{1 / 2}}{\sqrt{2} \pi \hbar^{3} \rho \omega_{j}}\left(e^{\frac{\hbar \omega_{j}}{k_{\beta} T}}-1\right)^{-1}\left\{\gamma_{i+n j}^{1 / 2} \gamma_{i+n j}^{\prime}+\gamma_{i-n j}^{1 / 2} \gamma_{i-n j}^{\prime} e^{\frac{\hbar \omega_{j}}{k_{\beta} T}}\right\}
\end{aligned}
$$




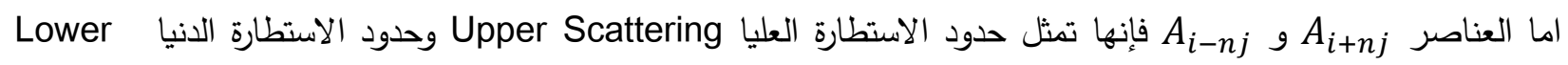
Scattering حدود الاستطارة وهي كميات متجه تقع على اقطار اعلى وادنى من الاقطار الرئيسة الثلاثة المصفوفة A، ويكون عدد هذه الحدود هو 15 لكل طاقة وبسبب خاصية التتاظر فان الحدود المتساوية تجمع قيمها مع بعضها فيصبح عددها 6 لكل استطارة كما مبين في المعادلات الاتية:

$A_{i+n j}=\sum_{j=1}^{15} \frac{D_{i v}^{2}\left(m_{t}^{2} m_{l}\right)^{1 / 2}}{\sqrt{2} \pi \hbar^{3} \rho \omega_{j}}\left(e^{\frac{\hbar \omega_{j}}{k_{\beta} T}}-1\right)^{-1}\left\{\gamma_{i+n j}^{1 / 2} \gamma_{i+n j}^{\prime} e^{\frac{\hbar \omega_{j}}{k_{\beta} T}}\right\}$

$A_{i-n j}=\sum_{j=1}^{15} \frac{D_{i v}^{2}\left(m_{t}^{2} m_{l}\right)^{1 / 2}}{\sqrt{2} \pi \hbar^{3} \rho \omega_{j}}\left(e^{\frac{\hbar \omega_{j}}{k_{\beta^{T}}^{T}}}-1\right)^{-1}\left\{\gamma_{i-n j}^{1 / 2} \gamma_{i-n j}^{\prime}\right\}$

يتم تمثيل المعادلة (18) بدلالة مصفوفات مربعة n ×n اذ يعتمد حجم المصفوفات على اختيار قيمة مقدار التغير في الطاقة ع وضمن المدى 2)eV - 0 ) فعندما تكون $0.001=000$ = 2000 ستكون كما مبين في المعادلة الاتية:

$n=\frac{2 \mathrm{eV}}{\Delta \varepsilon}$

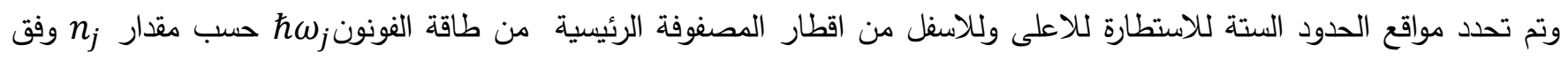
المعادلة الاتية:

$n_{j}=\frac{\hbar \omega_{j}}{\Delta \varepsilon}$

تعتمد طاقة الفونون على مقدار التغير في الطاقة عه الذي يؤدي الى تغيير في أبعاد المصفوفة A وكذلك الى تغيير في مواقع

حدود الاستطارة وبالتالي يؤدي الى تغيير في دقة الحسابات اضافة الى تغير سرعة تنفيذ هذه الحسابات مع تغيير ابعاد المصفوفة A. اذا

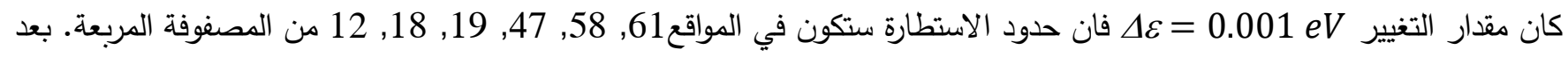
استخدام الشرط الحدودي 0 = (o) $f(1)$ وتقيم مناطق الطاقة الى 2000 نقطة للمنظومة اذ يمكن تمثل كل نقطة معادلة فروق مشابه

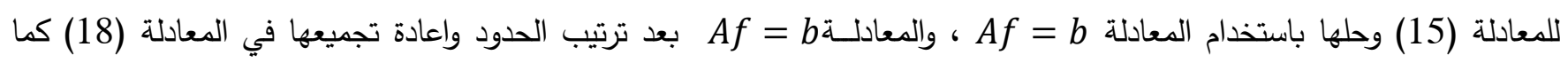
موضح في الثكل (1). 


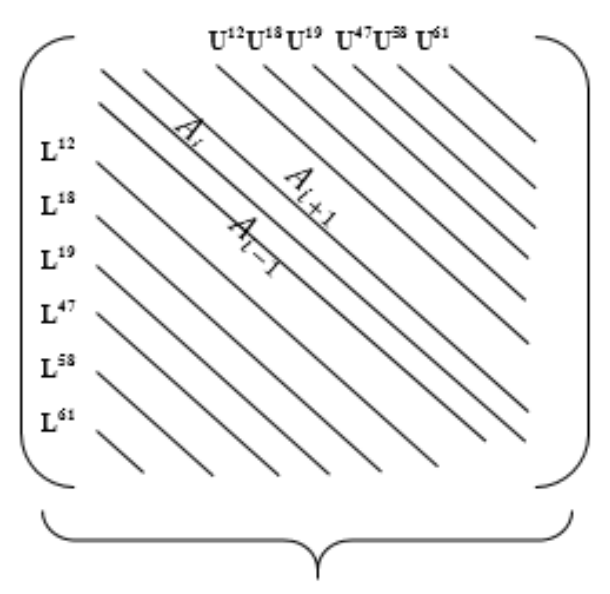

A

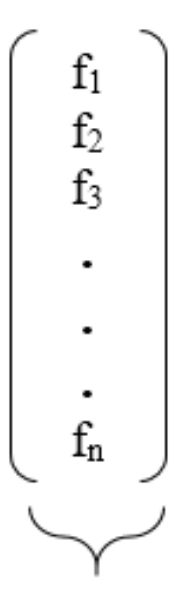

$\mathbf{f}=\mathbf{b}$

الثكل (1) : المعادلة الزيادة في الطاقة

اذا كان مقدار التغيير في الطاقة A الرئيسي في المصفوفة وتصبح الحدود 6 لكل استطارة 6,9,10,24,29,31 وعند الاستمرار بزيادة عه فانه يمكن لبعض حدود الاستطارة ان تشغل نفس الموقع وعليه يمكن جمع قيم عناصر لحدين ونضعهم في الموقع المحدد كما هي الحالة عندما تكون اذ تكون مواقع حدود الاستطارة 3,5,12,15 ويمكن ملاحظة أن مواقع حدود الاستطارة أصبحت أربعة مواقع والسبب اندماج عناصر الحد الثاني والثالث مع بعضهم واندماج عناصر الحد الخامس والسادس والثكل (2) يوضح مواقع حدود الاستطارة لقيم مختلفة للتغير في الطاقة 

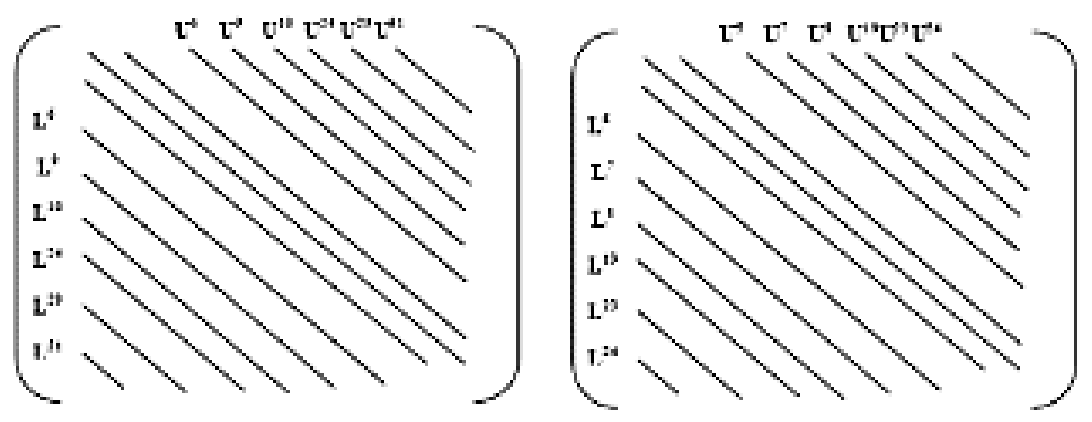

$$
\Delta \varepsilon=0.002 \quad n=1000
$$

$$
\Delta \varepsilon=0.0025 \quad n=800
$$
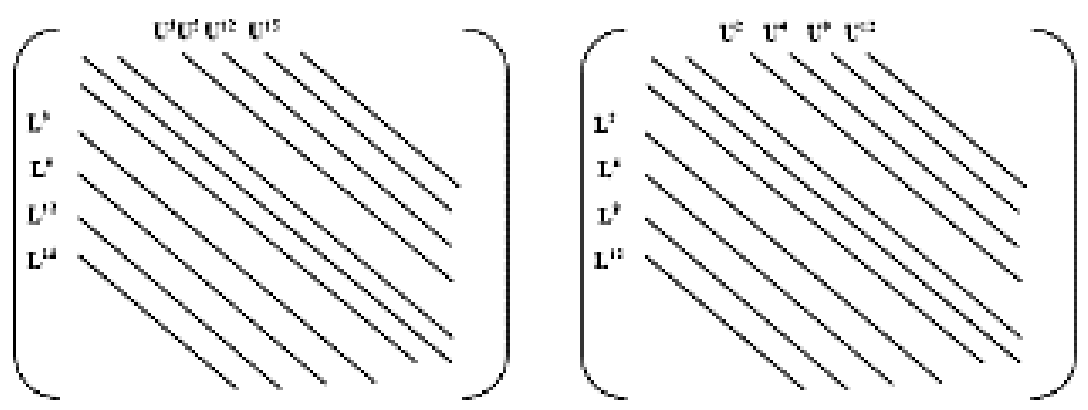

$$
\Delta \varepsilon=0.004 \quad n=500
$$

$$
\Delta \varepsilon=0.005 \quad n=400
$$
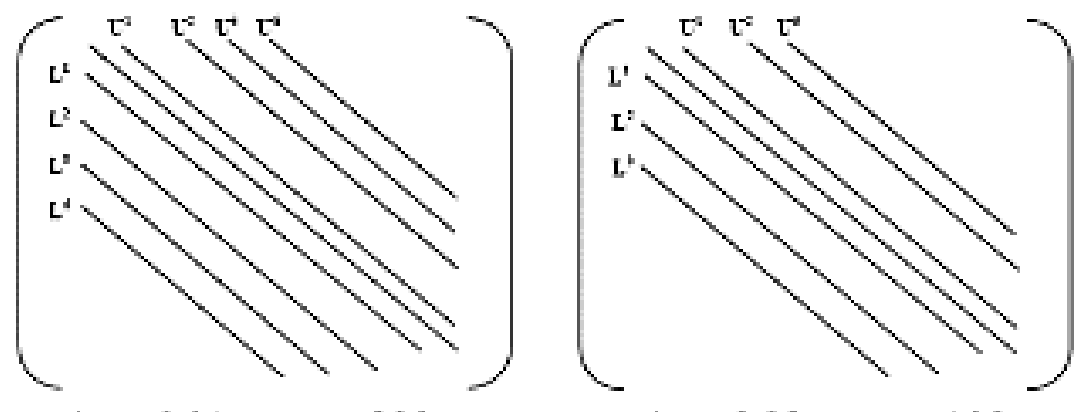

$$
\Delta \varepsilon=0.01 \quad n=200
$$

$$
\Delta \varepsilon=0.02 \quad n=100
$$

الثكل (2) : مواقع حدود الاستطارة في المصفوفة المربعة n× $n$ × تعتمد على مقدار التغيير في الطاقة عه في المصفوفة A للمنظومات لقيم مختلفة للتغير في الطاقة ع ه في المصفوفة A للمنظومات

عند حل المصفوفة Af =b عديا باستخدام طريقة الحذف لكاوس وحساب معامل لاجندر الاول يمكن حساب دالة توزيع الالكترونات وضربها بكثافة الحالات (ع) g) ومن ثم حساب معدل طاقة الالكترونات.

$g(\varepsilon)=\frac{\sqrt{2}}{\pi^{2} \hbar^{3}}\left(m_{x} m_{y} m_{z}\right)^{1 / 2} \gamma^{1 / 2}(\varepsilon) \gamma^{\prime}(\varepsilon)$

$$
I=\int_{0}^{\infty} f_{o}(\varepsilon) g(\varepsilon) d \varepsilon
$$

يتم معايرة الدالة باستخدام شرط العيارية الاتي: يبين الجدول (1) قيم مختلفة لعدد عناصر المصفوفة ولمدى متغير من الطاقة عه وقيم مواقع حدود الاستطارة العليا والدنيا 
n التي تعتمد على طاقة الفونون $n_{j}$ $<\varepsilon>=\int_{0}^{\infty} g(\varepsilon) \varepsilon f_{o}(\varepsilon) d \varepsilon$

تبين الخوارزمية (المخطط الانسيابي) المستخدمة في البحث طريقة حساب كل من كثافة الحالات ودالة التوزيع ومعدل طاقة الالكترون

كما موضح في الشكل (3)

جدول (1): مواقع حدود الاستطارة jn في المصفوفة المربعة A التي تعتمد على طاقة الفونون ولمدى متفير من الطاقة عه.

\begin{tabular}{|c|c|c|}
\hline $\boldsymbol{n}$ & $\Delta \boldsymbol{\varepsilon}$ & $n_{j}$ \\
\hline 100 & 0.02 & $1,2,3$ \\
\hline 200 & 0.01 & $1,2,5,6$ \\
\hline 400 & 0.005 & $2,4,9,12$ \\
\hline 500 & 0.004 & $3,5,12,15$ \\
\hline 1000 & 0.002 & $6,9,10,24,29,31$ \\
\hline 2000 & 0.001 & $12,18,19,47,58,61$ \\
\hline
\end{tabular}

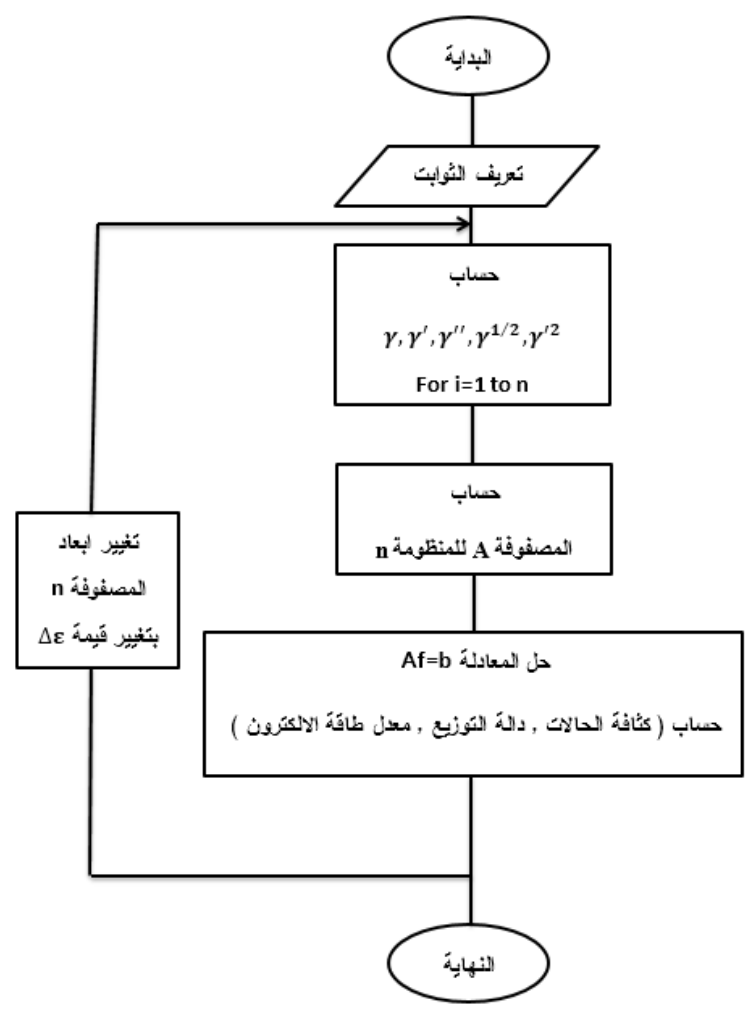

الثكل (3) مخطط الخوارزمية المستخدمة في البحث 
تم في هذا البحث حل المعادلة (18) عدديا باستخدام طريقة المصفوفة وتطبيق المعادلة Af =b وبتعويض قيم الثوابت الموضحة في جدول (2) وجدول (3) لست منظومات مختلفة تعتمد على مقدار التغير بالطاقة عه. كما هو مبين في الجدول (1)، تم اعداد برنامج بلغة MATLAB لحل معادلة بولتزمان شبه الكلاسيكية في السيلكون اذ يعتمد البرنامج على اختيار حجم المصفوفة A ل ان الزيادة في حجم المصفوفة A يؤدي الى زيادة في حجم الذاكرة وبالتالي زيادة وقت تتفيذ البرامج والحصول على الدقة في الحسابات. تم في لئي هذا العمل استقصاء الحجم الامثل للمصفوفة التي تعطي دقة في النتائج وتتوافق مع البحوث المنشورة. اذ تم اعتماد عدة قيم لثدة المجال الكهربائي المسلط ولمدى يمتد من المجالات الواطئة الى المجالات العالـية

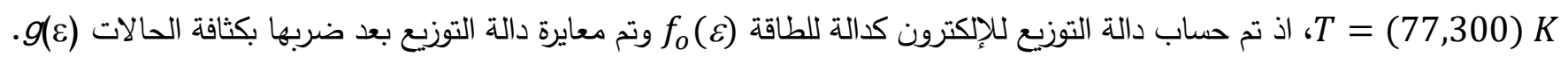
يمكن توضيح آلية حساب دالة التوزيع لبولتزمان في الثكل (3) الذي يمثل المخطط الانسيابي لحساب خواص المنظومات المستخدمة في

جدول (2): المعاملات الفيزيائية للسليكون [4,5,12].

\begin{tabular}{|l|c|c|c|}
\hline Parameter & Symbol & Value & Unit \\
\hline Mass density. & $\rho$ & 2329 & $\mathrm{Kg} / \mathrm{m}^{3}$ \\
\hline Non-parabolicity parameter. & $\alpha$ & 0.5 & $\mathrm{eV}^{-1}$ \\
\hline Electron acoustic deformation Potential. & $D_{\mathrm{ac}}$ & 9.5 & $\mathrm{eV}$ \\
\hline Free electron mass & $m_{0}$ & $9.1093897 \times 10^{-31}$ & $\mathrm{Kg}$ \\
\hline Longitudinal effective mass & $m_{l}$ & $0.98 m_{0}$ & $\mathrm{Kg}$ \\
\hline Transverse effective mass & $m_{t}$ & $0.19 m_{0}$ & $\mathrm{Kg}$ \\
\hline Reduced Planck constant & $\hbar$ & $6.582122025 \times 10^{-16}$ & $\mathrm{eV} . \mathrm{s}$ \\
\hline Boltzmann constant & $k_{\beta}$ & $8.61573 \times 10^{-5}$ & $\mathrm{eV} . \mathrm{k}^{-1}$ \\
\hline$f_{1}(T A)$ : equivalent energy, coupling constant & $\hbar \omega_{f_{1}}$ & 0.019 & $\mathrm{eV}$ \\
& $D_{f_{1}}$ & $0.3 \times 10^{10}$ & $\mathrm{eV} / \mathrm{m}$ \\
\hline$f_{2}(L A)$ : equivalent energy, coupling constant & $\hbar \omega_{f_{2}}$ & 0.047 & $\mathrm{eV}$ \\
& $D_{f_{2}}$ & $2 \times 10^{10}$ & $\mathrm{eV} / \mathrm{m}$ \\
\hline$f_{3}(T O)$ : equivalent energy, coupling constant & $\hbar \omega_{f_{3}}$ & 0.058 & $\mathrm{eV}$ \\
& $D_{f_{3}}$ & $2 \times 10^{10}$ & $\mathrm{eV} / \mathrm{m}$ \\
\hline$g_{1}(T A)$ : equivalent energy, coupling constant & $\hbar \omega_{g_{1}}$ & 0.012 & $\mathrm{eV}$ \\
& $D_{g_{1}}$ & $0.5 \times 10^{10}$ & $\mathrm{eV} / \mathrm{m}$ \\
\hline$g_{2}(L A)$ : equivalent energy, coupling constant & $\hbar \omega_{g_{2}}$ & 0.018 & $\mathrm{eV}$ \\
& $D_{g_{2}}$ & $0.8 \times 10^{10}$ & $\mathrm{eV} / \mathrm{m}$ \\
\hline$g_{3}(L O)$ : equivalent energy, coupling constant & $\hbar \omega_{g_{3}}$ & 0.061 & $\mathrm{eV}$ \\
& $D_{g_{3}}$ & $11 \times 10^{10}$ & $\mathrm{eV} / \mathrm{m}$ \\
\hline
\end{tabular}

جدول (3) قيم المعامل $\tau_{o}$ مع مديات الطاقة ولارجة العرارة T= 77, 300 [12].

\begin{tabular}{|c|c|c|}
\hline Lattice Temperature K & Electron energy $\boldsymbol{e V}$ & $\boldsymbol{\tau}_{\boldsymbol{o}}\left(\boldsymbol{e V}^{\mathbf{1 / 2}} \boldsymbol{s}\right)$ \\
\hline \multirow{2}{*}{$\mathbf{3 0 0}$} & $4.07 \times 10^{-14}$ & $0<\varepsilon<0.06$ \\
& $2.03 \times 10^{-14}$ & $0.06<\varepsilon<2$ \\
\multirow{2}{*}{$\mathbf{7 7}$} & $19.00 \times 10^{-14}$ & $0<\varepsilon<0.03$ \\
& $3.43 \times 10^{-14}$ & $0.03<\varepsilon<2$ \\
\hline
\end{tabular}




\section{Journal of Education and Science (ISSN 1812-125X), Vol: 29, No: 4, 2020 (108-123)}

يبين الثكل (4) دالة التوزيع كدالة للطاقة عند قيم متعددة للمجال الكهربائي اذ ان لكل قيمة للمجال الكهربائي قيمة عظمى لدالة التوزيع عند طاقة معينة واعلى قيمة لدالة التوزيع كانت عند المجالات الواطئة $10 \mathrm{kV} / \mathrm{cm}$ عند الطاقة $0.024 \mathrm{eV}$ لدرجة الحرارة

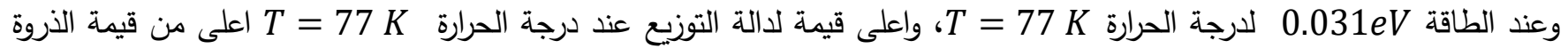
للتوزيع عند درجة الحرارة K وتتسع مع زيادة الطاقة وذلك بسبب زيادة القوة المعلة للالكترونات بسبب زيادة المجال المسلط، اي ان التوزيع يزاح باتجاه الطاقات الاعلى وقمة دالة التوزيع تزاح قليلا باتجاه الطاقات العالية، ودهاه

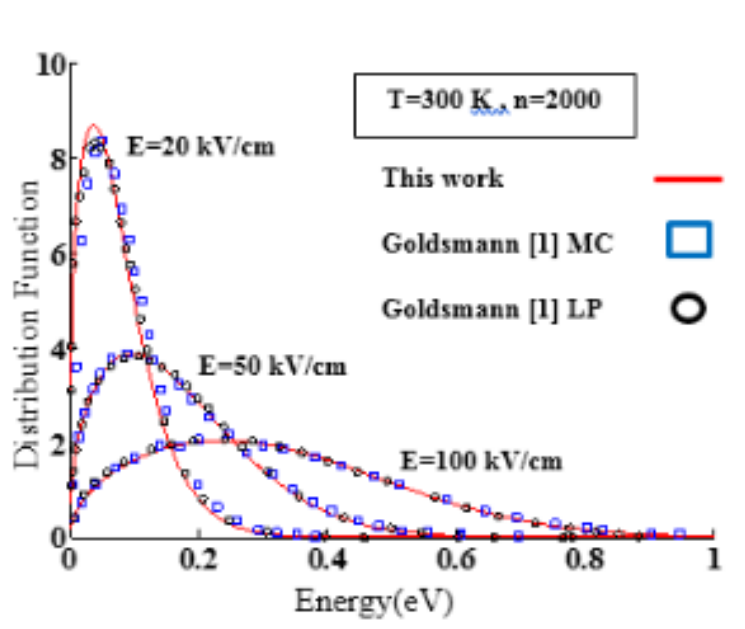

(a)

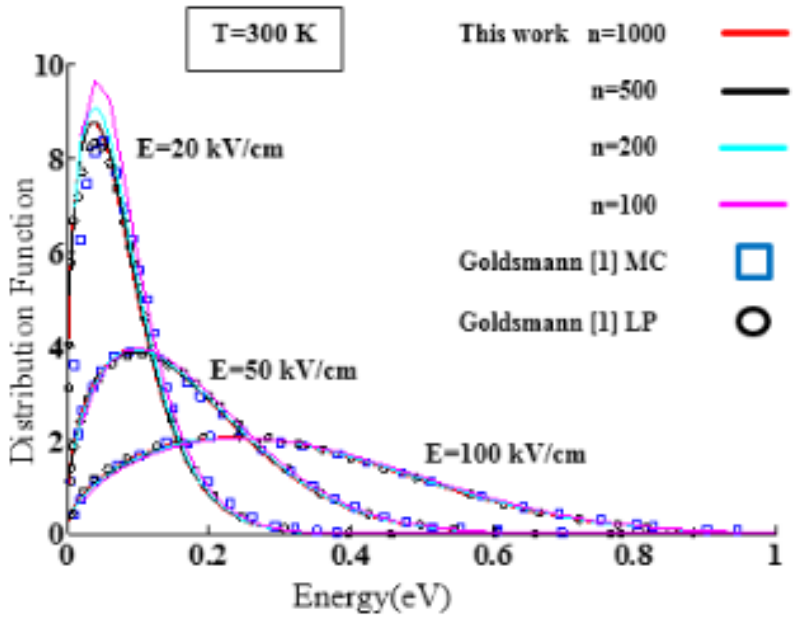

(b)

الثكل (4): العلاقة بين الطاقة و دالة توزيع الاكترون في السليكون للمنظومة 2000 × 2000 ولمدى من المجالات الكهبائية وعند درجتي الحرارة 77 و $.300 K$ و

وتكون على مدى واسع من قيم الطاقة وهذا يعني ان طاقة الالكترونات ستكون ضمن مدى اوسع من قيم الطاقة. يمكن ملاحظة ان ذروة دالة التوزيع عند درجة الحرارة T=300 التوزيع عند درجة الحرارة 77 اكثر من ازاحة دالة التوزيع عند درجة الحراة المجالات الكهربائية العالية.

من مقارنة نتائج المنظومات المستخدمة في هذا البحث مع نتائج البحوث المنشورة التي تم حساب دالة التوزيع بطريقتي مونتي كارلو

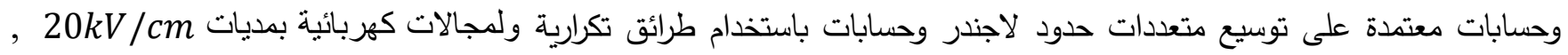

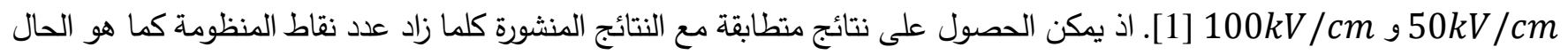
في المنظومة المربعة 2000×2000 عندما يكون مقدار التغيير في الطاقة $0.001=0$ فيها عدد النقاط مثل فان ذروة دالة التوزيع تكون مزاحة عن النتائج المنشورة كما هو الحال في المنظومات 100×100 و 200×200 لاحظ الثكل (5b). (15). 
تم حساب معدل طاقة الاككترون كدالة للمجال الكهربائي ولكافة المنظومات المستخدمة عند درجة حرارة 300K اذ بينت النتائج تطابقا ولكافة المنظومات في منطقة المجالات العالية وانحراف قليل عند المجالات الكهربائية الواطئة كما مبين في الثكل (6).
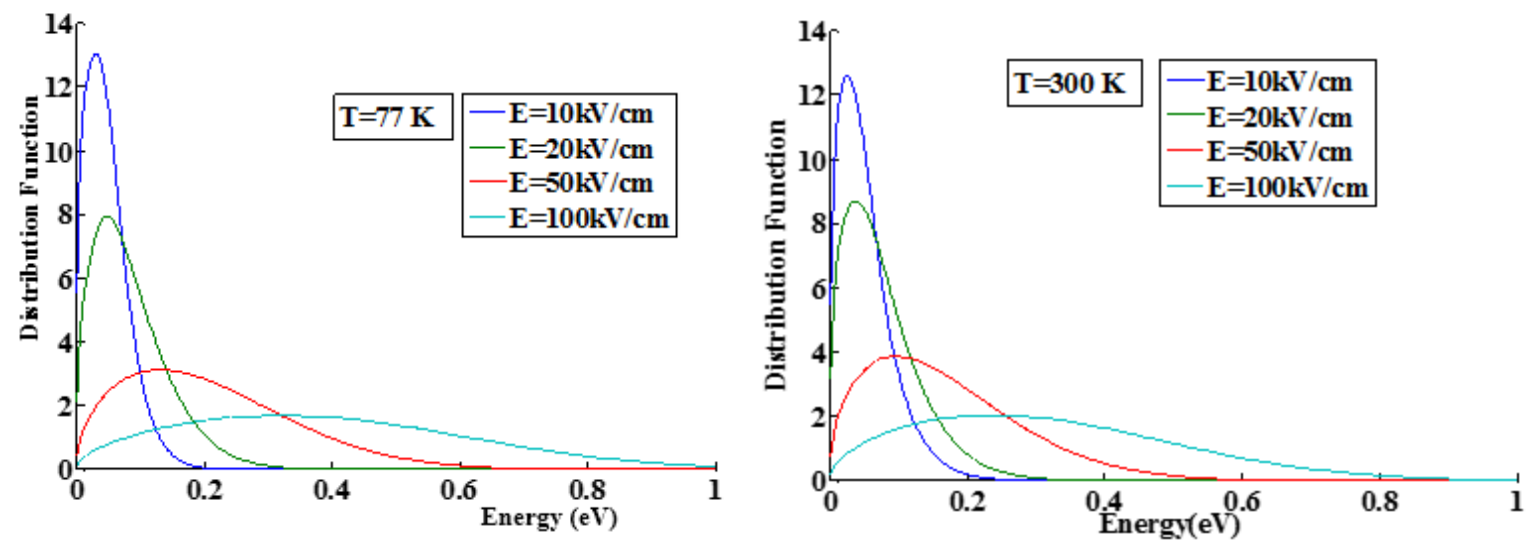

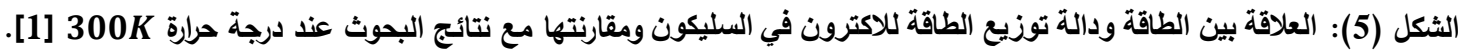

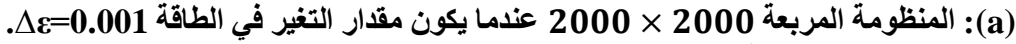

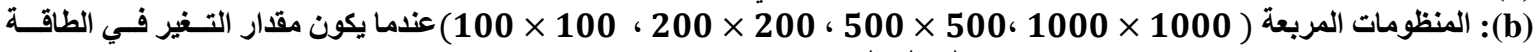
( على التوالي. $0.02,0.01,0.004,0.002)$

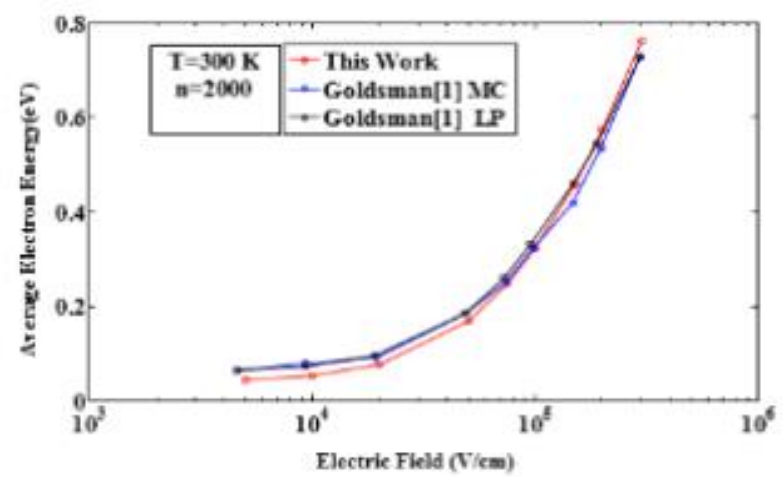

(a)

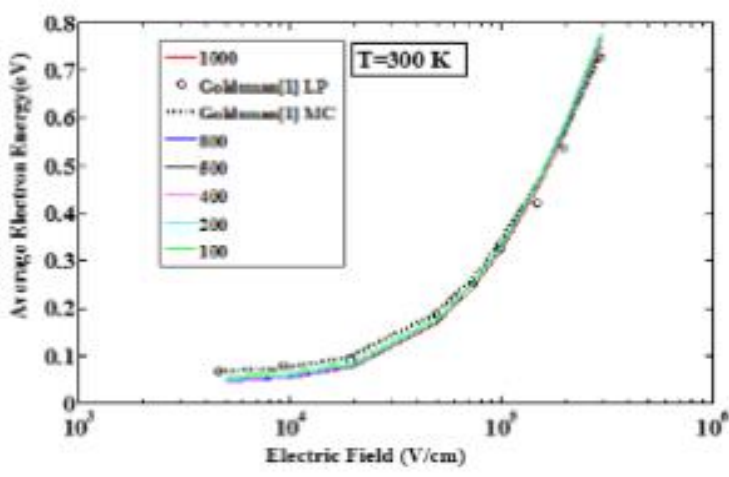

(b)

الثكل (6): معدل الطاقة للإلكترون كدالة لثدة المجال الكهبائي في السليكون ومقارنتها مع نتائج البحوث عند درجة حرارة 300 [1]].

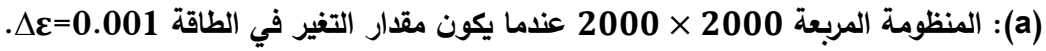

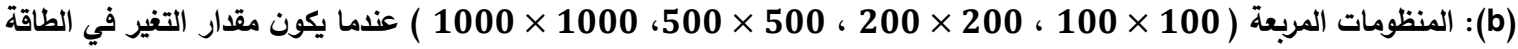

$$
\text { (التى }=0.02,0.01,0.004,0.002)
$$

تم تقييم اداء البرنامج الذي أعد والحسابات للمنظومات المستخدمة عن طريق حساب الزمن المستغرق لتتفيذ حسابات دالة التوزيع مع زيادة أبعاد المنظومة كما هو في الثكل (7) اذ إن الزمن المستغرق في حساب دالة التوزيع يزداد بثكل اسي وخاصة عند المنظومة 2000 × 2000 التي استغرقت 16 ثانية تقريبا للحساب في حين ينخفض الوقت للمنظومة 1000 × 1000 الى 4 ثانية تقريبا. 


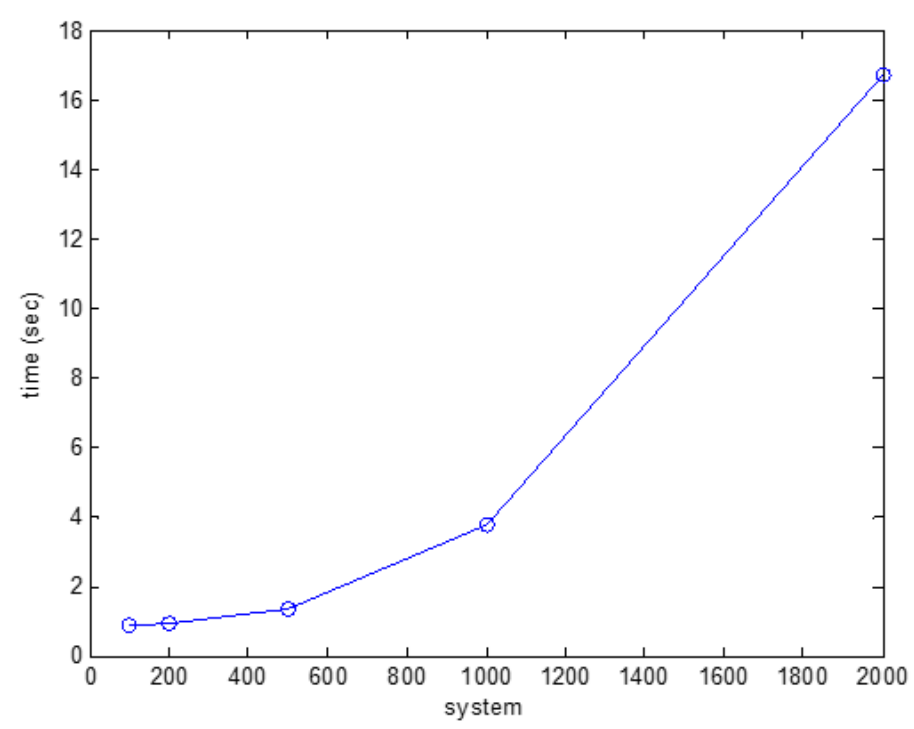

الثكل (7): أبعاد المنظومة n= 100, 200, 500, 1000, and 2000 على التوالي كدالة للزمن المستغرق في حساب دالة التوزيع. الاستنتاجات:

تم تصميم عدة منظومات لاختيار الحجم المناسب للمصفوفة في حل معادلة بولتزمان شبه الكلاسيكية في السيلكون والحصول على الدقة المناسبة. اذ بينت النتائج ان الاختيار الامثل لحجم المصفوفة يكون بين توافق مع النتائج المنشورة عند المجالات العالية والواطئة. اما عند قيم المجالات العالية فلا يثكل حجم المصفوفة تغيرات كبيرة في النتائج التي يتم الحصول عليها. تبتعد النتائج عن الدقة لقيم 500 > $n$ للمجالات الكربائية التي تقل عن 10kV/cm. وتتاثر معدل طاقة الاككترونات كثيرا بزيادة المجال المسلط وتتوافق نتائج البحث للمنظومات المختلفة مع النتائج المنشورة، تتاثر دالة التوزيع في المنظومات المختلفة المستخدمة في البحث بدرجة كبيرة بتغير شدة المجال المسلط وتتاثر بدرجة اقل بتغيرات درجة الحرارة، الوقت المستغرق لتنفيذ البرنامج يزداد بشكل ملحوظ عند تجاوز 1000 n في حين لاتتاثر دقة الحسابات كثيرا بهذه الزيادة.

شكر و تقدير

يتقدم الباحثان بالثكر والتقدير لعمادة كلية التربية للعلوم الصرفة وقسم الفيوياء على دعم البحث وكذلك جزيل الثكر والعرفان للدكتور على عباس من قسم الفيزياء كلية التزبية للعلوم الصرفة جامعة الموصل و الدكتور موفق خليل احمد من قسم الفيزياء كلية التربية للعلوم الصرفة جامعة صلاح الدين/ اربيل للملاحظات القيمة التي اغنت البحث.

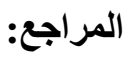

1-Goldsman, N., Henrickson, L., and Frey, J., Solid-State Electronics, 34(4), 389-396, (1991).

2- Shockley W., Solid State Electronics, 2,35, (1961)

3-Frey, J., \& Goldsman, N., IEEE Electron Device Letters, 6(1), 28-30, (1985). 
4- Lundstrom M. "Fundamentals of Carrier Transport". 2 2d Ed, Cambridge (2000).

5- Jacoboni, C., \& Reggiani, L., Reviews of Modern Physics, 55(3), 645, (1983).

6- Fischetti, M. V., \& Laux, S. E., Physical Review B, 38(14), 9721, (1988).

7- Pop, E., Dutton, R. W., \& Goodson, K. E., Journal of Applied Physics, 96(9), 4998-5005, (2004).

8- Goldsman, N., Wu, Y. J., \& Frey, J., Journal of Applied Physics, 68(3), 1075-1081, (1990).

9- Muscato, O., \& Di Stefano, V., Journal of Computational Electronics, 11(1), 45-55, (2012).

10- Castiglione, T., \& Muscato, O., Journal of Computational and Theoretical Transport, 46(3), 186-201, (2017).

11- Mohammad Salih, Ali Abbas, PhD. Thesis, College of Science, University of Mosul (in Arabic), (2010).

12- Ahmed, Moufaq Jalil, PhD. Thesis, College of Science, University of Salahaddin, (2007).

13- Scherer, Philipp OJ. Computational Physics. Springer Berlin Heidelberg, (2010). 